\title{
地理生态学的干燥度指数及其应用评述
}

\author{
孟 猛 ${ }^{1}$ 倪 健 $^{2 *} \quad$ 张治国 ${ }^{1}$
}

(1 山东大学生命科学学院, 济南 250100) (2 中国科学院植物研究所植被数量生态学重点实验室, 北京 100093)

\begin{abstract}
摘 要 干燥度指数 (Aridity index, $A I$, 该文特指气候干燥度) 是表征一个地区干湿程度的指标, 在地理学和生态学 研究中长期应用, 近来成为全球变化研究中经常涉及到的气候指标之一, 尤其是气候变化和干旱化、荒漠化等研 究。该文列举了国内外目前存在的 22 种干燥度指数, 并对目前常用的 8 种指数进行了详细描述和分析, 包括各自 的原理、计算方法和在生态学与地理学研究中的应用等。结合其应用和中国的实际状况, 分析了各自的优缺点, 认 为修改后的谢氏干燥度、de Martonne 干燥度和 Holdridge 可能蒸散率(等同于某种意义上的干燥度)计算方法简单实 用, 有着明确的物理学和生态学意义, 与我国的实际情况对应性较强, 适合于中国气候变化及其相关的干旱化、荒 漠化等方面的研究。
\end{abstract}

关键词 干燥度 可能蒸散量 气候变化 气候区划 干旱化 荒漠化

\section{ARIDITY INDEX AND ITS APPLICATIONS IN GEO-ECOLOGICAL STUDY}

\author{
MENG Meng ${ }^{1}$ NI Jian ${ }^{2 *}$ and ZHANG Zhi-Guo ${ }^{1}$ \\ (1 School of Life Sciences of Shandong University, Jinan 250100 , China) \\ (2 Laboratory of Quantitative Vegetation Ecology , Institute of Botany , Chinese Academy of Sciences , Beijing 100093 , China)
}

\begin{abstract}
The aridity Index ( $A I$, here defined as the climatic aridity) is an index that describes the dry and wet conditions of a site. Historically, the $A I$ has been often used in long-term studies of geography and ecology . Recently it has became one of the most frequently used climatic factors in studies of global change , especially in studies of climate change, ariditification and desertification. A total of $22 \mathrm{AI}$ s used worldwide were briefly introduced in this paper, and of these eight of the more commonly used $A I \mathrm{~s}$ were evaluated. We discussed their principles, calculation methods, their applications in ecological and geographical studies, and the advantages and shortcomings of each indice were analyzed based on their applications and practice in China. Our analyses indicated that three $A I \mathrm{~s}$, the modified Selianinov $A I$, the de Martonne $A I$ and the Holdridge potential evapotranspiration ratio (another $A I$ to some extent) have clear applicability and significance for physics and ecology. These $A I \mathrm{~s}$ are suitable for characterizing the physical environment of China and can be used in future studies of climate change, aridification and desertification in China.
\end{abstract}

Key words Aridity index , Potential evapotranspiration , Climatic change , Climate regionalization , Ariditification , Desertification

干燥度指数 (Aridity index, $A I$ 或其它缩写形式, 如 $K$ 等)，在此特指气候干燥度, 是表征一个地区干 湿程度的指标，一般以某个地区水分收支与热量平 衡的比值来表示, 其倒数称为湿润指数 (Humidity in$\operatorname{dex}, H I)$ 。

中外学者自 1900 年以来提出了许多计算干燥 度指数的方法, 简单的是以经验公式如降水和气温 的比值来表达,复杂的则是通过计算可能蒸散量 (Potential evapotranspiration, $P E$ ) ,以降水与可能蒸散
的对比关系获得干燥度。这些计算方法的原理各 异, 各有优缺点, 并应用于不同的地区和不同的研究 中。由于干燥度指数计算原理和方法的差异以及使 用地区和应用目的的不同, 造成了其指标范围也各 自不同, 使得不同干燥度指数的比较存在较大困难, 其应用如干湿气候区划也存在较大差异和不可比 性，因而造成了一定的混乱。

当前, 气候变化是全球环境变化研究的一个重 要领域,尤其是在中纬度的干旱-半干旱地区,干旱 
化、荒漠化等与气候变化息息相关的诸多研究, 都与 干燥度指数的计算和表达存在着千丝万缕的联系。 但由于过去工作中干燥度指数计算方法的差异, 使 得许多工作难以比较, 无法把握气候变化的准确信 息, 并在较大空间和较长时间尺度上统一量化和应 用。因此, 本文对目前常用的干燥度指数进行比较, 结合各干燥度指数的应用, 分析其优缺点, 并选择简 单实用、物理学和生态学意义明确的干燥度指数, 作 为今后我国全球变化研究的干湿气候因子分析的参 数。这对于了解气候-植被之间的相互关系, 研究和 预测全球变化对人类及生物赖以生存的生态环境的 影响，以及制定应对的策略、方法与途径具有重要的 理论和现实意义; 同时, 干燥度指数的统一和可比 性，对于我国干湿气候区划、荒漠化、干旱化等相关 研究会起到重要的作用。

\section{1 现行干燥度指数概述}

自 1900 年以来, 中外学者陆续提出了 22 种干 燥度 (或湿润指数) 的计算方法, 根据资料 (Dzerdzeevskii, 1958; 耿宽宏, 1986; 倪健, 1998; 黄培祐， 2002)整理, 主要方法见表 1 。

\section{2 几种常用的干燥度指数计算方法与应用}

表 1 分析了 22 种干燥度指数的计算原理和方 法 结合其应用, 我们选择以下几种干燥度指数进行 详细论述, 并按照其水分收支计算方法的差异 划分 为几种干燥度类型。

\section{1 降水量作为干燥度的代用指标}

最简单直接的干燥度计算, 是以多年平均降水 量 (Precipitation, $P$ ) 作为标准。联合国粮农组织 (Food and agriculture organization, FAO) 认为, 农作物 及草地生产潜力, 或者作物种类、耕作制度以及草地 管理方式的选择, 取决于降水及土壤水分对植物或 作物生长期的有效性, 该生长期的降水量是气候区 划的重要指标(陈建伟等,1996)。在我国以年降水 量为指标划分干湿气候区存在 3 种不同的意见:1) 年降水量小于 $200 \mathrm{~mm}$ 为干旱区, $200 \sim 400 \mathrm{~mm}$ 为半 干旱蛆 ;2) 年降水量大于 $200 \mathrm{~mm}$ 为干旱区 ,200 $450 \mathrm{~mm}$ 为半干旱区 ;3) 年降水量小于 $250 \mathrm{~mm}$ 为干 旱区, 250 $500 \mathrm{~mm}$ 为半干旱区。任福民等 (1995)利 用全国 160 个气象站 1951 1992 年的年降水标准化 资料，以年降水 $700 \mathrm{~mm}$ 为标准，将全国划分为两个 大区 :干旱半干旱区和非干旱区。由此可见,干旱与 非干旱的差别，在年平均降水量上可表达为 $400 、 500$
和 $700 \mathrm{~mm} 3$ 个标准。

此类以降水量作为干燥度代用指标的方法不仅 在具体划分的标准上存在分歧，而且只考虑了水分 收入，未考虑水分的支出，没有水分平衡的概念，不 能定量说明水分的盈亏,无法如实反映自然界的真 实面貌, 特别是植物与土壤的分布,生态学意义不明 确。因此，降水量指标作为干燥度指数的代用品，仅 可在其它气象资料缺乏而只有降水量数据存在时使 用, 如果有较丰富的其它气候指标, 则需要配合这些 指标使用。

\section{2 可能蒸散量计算的干燥度}

按 Penman(1948)的定义, 潜在蒸发是 从不匮缺 水分的、高度一致并全面遮覆地表的矮小绿色植物 群体在单位时间内的蒸腾量”,包括从所有表面的蒸 发与植物蒸腾。可能蒸散量 $(P E)$ 与降水量 $(P)$ 之 比即干燥度 $(K)$, 计算公式如下:

\section{$K=P E / P$}

目前，以可能蒸散量计算干燥度的方法在国际 上比较流行。按照计算可能蒸散量的方法不同, 可 以分为 Penman 和 Thornthwaite 方法以及目前在国内 外应用较多的 Holdridge 生命地带分类系统中的可 能蒸散计算方法。

\subsubsection{Penman 的可能蒸散量}

Penman 公式法(Penman ,1956)由 Penman 在 1948 年提出, 并在 1956 年对其指标系统进行了部分改 进。

$$
E_{0}=\frac{\Delta H+\gamma E_{a}}{\Delta+\gamma}
$$

式中, $H$ 为净辐射量 ; $\Delta$ 为气温 $T_{a}$ 时的饱和水汽压 曲线的斜率; $\gamma$ 为干湿表常数 $\left(0.46 \mathrm{~mm} /{ }^{\circ} \mathrm{C}\right) ; E_{a}$ 为 干燥力。净辐射量

$$
H=R_{A}(1-a)\left(0.18+0.55 \frac{n}{N}\right)-\sigma T^{4}(0.56-
$$

$\left.0.99 \sqrt{e_{d}}\right)\left(0.10+0.90 \frac{n}{N}\right)$

式中, $R_{A}$ 为太阳辐射的理论最大值; $a$ 为发射率; $n / N$ 为日照百分率; $\sigma T^{4}$ 为气温 $T$ 时的理论黑体辐 射 $; e_{d}$ 为水汽压。

$$
E_{a}=0.35\left(1+\frac{u}{100}\right)\left(e_{a}-e_{d}\right)
$$

式中, $u$ 为 $2 \mathrm{~m}$ 高度的风速; $E_{a}$ 为空气的饱和水汽 压; $\left(e_{a}-e_{d}\right)$ 为百叶箱高度的饱和差。

席承藩等 (1984) 在进行中国自然区划时，对干 湿状况的划分是用 Penman 公式计算的年干燥度, 并 参考年降水量作为指标。张新时 (1989a; ;1989b) 提 


\section{表 1 干燥度指数(或湿润指数)计算方法}

Table 1 The formulas of aridity index (or humidity index) ( $A I$ )

\begin{tabular}{cccc}
\hline 年份 & 创建者 & 表达式 & 备注 \\
Year & Establisher & Formula & Note \\
\hline
\end{tabular}

$1900 \quad$ V. V. Dokutchaev $\frac{P}{E_{0}}$

把多年平均年降水量 $(P)$ 与多年平均年水平蒸发量 $\left(E_{0}\right)$ 进行比 较, 定性分析干燥度 To compare the mean annual precipitation $(P)$ with mean annual evaporation $\left(E_{0}\right)$ and then to analyze the aridity index

1905 G. N. Vyssotsky

1911

E. M. Oldekop

$\frac{P}{E_{0}}$
干燥度的准确计算 The accurate calculation of aridity index

可能蒸散量 $E_{0}$ 的第一次应用, $E_{0}=a d, a$ 为湖面水平面蒸发与气 象数据比较所得系数, $d$ 为饱和差。实际公式可表示为 $: \frac{P}{232 d}$

This was the first use of potential evapotranspiration $\left(E_{0}\right) . E_{0}=a d$, where $a$ is a coefficient of comparison between evaporation of horizontal lake level and meteorological data ; $d$ is the saturation deficiency. The equation expresses as $\frac{P}{232 d}$

$P$ 为年平均降水量, $t^{0}$ 为年平均温度 $P$ is the mean annual precipitation $; t^{0}$ is the mean annual temperature

同上 Idem

$\begin{array}{llll}1922 & \text { W. Köppen } & \frac{P}{2\left(t^{0}+7\right)} & \text { 同上 Idem } \\ 1925 & \text { E. de Martonne } & \frac{P}{t^{0}+10} & \text { 同上 Idem }\end{array}$

\begin{tabular}{clcl}
\hline 1926 & A. Meyer & $\frac{P}{d}$ & $d$ 为饱和差 $d$ is the saturation deficiency \\
\hline 1928 & E. Reichel & $\frac{N P}{t^{0}+10}$ & $N$ 为降水天数 $N$ is the number of days of rainfall \\
\hline 1932 & V. B. Shostakovitch & $\frac{P}{t^{0}+10}$ & $\begin{array}{l}t^{0} \text { 为植被生长期平均温度 } t^{0} \text { is the mean temperature of the period for } \\
\text { vegetation growth }\end{array}$ \\
\hline
\end{tabular}

1939 L. Emberger

$\overline{(M+m)(M-m)} \quad$ maximum temperature of the warmest month, $m$ is the mean minimum temperature of the coldest month

1937 G. T. Selianinov $\quad 0.1 \frac{\sum t^{0}}{P}$

$\sum t^{0}$ 为日均温大于 $10{ }^{\circ} \mathrm{C}$ 的年积温 $\sum t^{0}$ is the annual accumulated temperature that the daily mean temperature is larger than $10{ }^{\circ} \mathrm{C}$

$E_{0}$ 为可能蒸散量, $E_{0}=0.0018\left(25+t^{0}\right)^{2}(100-a), t^{0}$ 为月均温, ture, $a$ is the monthly mean relative humidity

\begin{tabular}{|c|c|c|c|}
\hline 1945 & T. Kira & $\begin{array}{l}\text { 当 } W I=0 \sim 100{ }^{\circ} \mathrm{C} \cdot \text { 月 }{ }^{-1} \text { 时 }, k= \\
P /(W I+20) \text {; 当 } W I>100{ }^{\circ} \mathrm{C} . \\
\text { 月 }{ }^{-1} \text { 时 }, k=2 P /(W I+140)\end{array}$ & $\begin{array}{l}k \text { 为干湿度指数, WI 为温暖指数 } k \text { is the aridity-humidity index } \\
\text { (moisture index) ,WI is the warmth index }\end{array}$ \\
\hline 1947 & N. V. Bova & $\frac{H+P}{\sum t^{0}}$ & $H$ 为最初土壤湿润值 $H$ is the early value of soil moisture \\
\hline 1947 & L. R. Holdridge & $\frac{P E}{P}$ & $\begin{array}{l}P E \text { 为 Holdridge 公式计算的可能蒸散量 } P E \text { is the Holdridge' s po- } \\
\text { tential evapotranspiration }\end{array}$ \\
\hline 1948 & H. L. Penman & $\frac{P E}{P}$ & $\begin{array}{l}P E \text { 为 Penman 公式计算的可能蒸散量 } P E \text { is the Penman's potential } \\
\text { evapotranspiration }\end{array}$ \\
\hline 1948 & C. W. Thornthwaite & $\frac{P E}{P}$ & $\begin{array}{l}P E \text { 为 Thornthwaite 公式计算的可能蒸散量 } P E \text { is the Thornthwaite' } \mathrm{s} \\
\text { potential evapotranspiration }\end{array}$ \\
\hline 1949 & J. A. Prescott & $\frac{P}{(S d)^{0.7}}$ & $S d$ 为饱和差 $S d$ is the saturation deficiency \\
\hline 1950 & A. A. Skvortsov & $\frac{E_{a}}{E_{s t}}$ & $\begin{array}{l}E_{a} \text { 为实际蒸发 }, E_{s t} \text { 为标准蒸发 } E_{a} \text { is the actual evaporation, } E_{s t} \text { is the } \\
\text { standard evaporation }\end{array}$ \\
\hline
\end{tabular}


表 1 (续) Table 1 (continued)

\begin{tabular}{|c|c|c|c|}
\hline $\begin{array}{l}\text { 年份 } \\
\text { Year }\end{array}$ & $\begin{array}{c}\text { 创建者 } \\
\text { Establisher }\end{array}$ & $\begin{array}{l}\text { 表达式 } \\
\text { Formula }\end{array}$ & $\begin{array}{l}\text { 备注 } \\
\text { Note }\end{array}$ \\
\hline 1951 & M. J. Budyko & $R / L P$ & $\begin{array}{l}R \text { 为太阳净辐射, } L \text { 为蒸发潜热 } R \text { is the solar net radiation, } L \text { is the } \\
\text { evaporation latent heat }\end{array}$ \\
\hline 1952 & S. J. Kostin & $\frac{P}{E_{0}}$ & $\begin{array}{l}E_{0} \text { 为可能蒸散量, 计算公式为 } E_{0}=\frac{d n}{4}(1+0.004 t)^{2}, d \text { 为干旱期 } \\
\text { 水分不足量 }, n \text { 为植物生长水分需要量 } E_{0} \text { is the potential evapotran- } \\
\text { spiration : } E_{0}=\frac{d n}{4}(1+0.004 t)^{2} \text {, where } d \text { is the water deficiency dur- } \\
\text { ing the dry period ; } n \text { is the water demand of plant growth }\end{array}$ \\
\hline 1959 & 张宝 & $0.16 \frac{\text { 全年 } \geq 10^{\circ} \mathrm{C} \text { 的积温 }}{\text { 全年 } \geq 10^{\circ} \mathrm{C} \text { 期间的降水量 }}$ & $\begin{array}{l}\text { 将谢良尼诺夫公式根据中国实际情况修改所得 Based on the modi- } \\
\text { fication of the Selianinov's equation according to the actual situation in } \\
\text { China }\end{array}$ \\
\hline
\end{tabular}

供了 Penman 公式法的计算机计算与分类程序, 使复 杂的计算简单化，便于使用。倪健 (1998) 根据中国 亚热带常绿阔叶林区域的气象资料和植物分布资 料, 计算了组成中国亚热带常绿阔叶林 14 个主要科 112 个优势种及常见种的 Penman 可能蒸散值和干 燥度值, 按气候指标的分布范围将优势种和常见种 划分了 5 个水热分布类群。Liu 等 (1998) 在利用气 象和植被数据研究我国东北地区的生命地带变化 时,使用了 Penman 公式法来计算可能蒸散量。 Shang 等 (2001a) 在研究我国水分状况的数字生态模 型中也用此方法计算可能蒸散量。Shang 等 (2001b) 在研究我国的气象信息系统中利用 Penman 公式计 算了全国范围的干燥度, 并根据计算结果作出了数 字化地图。周晓东等 (2002) 在同一气候资料的基础 上, 分别应用 Thornthwaite 公式和 Penman 公式计算 了潜在蒸散, 并据此计算湿润指数 划分了我国的荒 漠化气候类型区, 确定了荒漠化潜在发生范围，同时 对上述两种方法在我国荒漠化气候类型区划中的适 用性进行了比较分析, 认为对我国而言, Penman 公 式法比 Thornthwaite 公式法更具有合理性。

1977 年在肯尼亚召开的联合国沙漠化会议上 曾散发了以 Penman 湿润指数为基础的世界干燥区 域分布图。Henry (1996) 用此计算方法对全球气温 升高后的可能蒸散量的变化进行了预测, 他认为气 温每升高 $1{ }^{\circ} \mathrm{C}$, 可能蒸散量就增加 $5.25 \%$ 。Hess (1998) 在尼日利亚东北部的干旱区研究中利用多种 方法对 $1961 \sim 1990$ 年的气象数据进行分析, 认为 Penman 方法与实际情况最为符合, 特别是以每月的 气象数据进行计算时。

Penman 公式是从能量平衡和空气动力学理论 出发建立起来的, 它采用水汽压、净辐射和在一定温
度条件下的空气干燥力以及风速来确定潜在蒸散, 具有坚实的理论基础和明确的物理意义，因而不能 算作是一个经验公式(张新时,1989a；1989b)。但同 时此方法所需气候指标较多，计算复杂，而且一些参 数如水汽压亏损值等在一般台站没有记录, 在实际 应用中还是受到一定的限制。所以在我国不能得到 广泛的应用。

\subsubsection{Thornthwaite 的可能蒸散量}

Thornthwaite 方法 ( Thornthwaite ,1948) 也是一种 国际上通用的计算可能蒸散量的方法, 被广泛应用 于气候分类和植被-气候关系研究。Thornthwaite 公 式法不仅给出了计算可能蒸散量的公式, 还进一步 根据月平均降水量与可能蒸散的差值进行土壤水分 平衡的计算，从而对估量各种土壤条件下的作物灌 溉需水量有参考价值。其公式为 :

$$
P E=\sum_{i=1}^{12} k_{i} e_{i}
$$

式中, $k_{i}$ 为第 $i$ 月的标准月换算因子, 它主要随纬度 不同而异。Thornthwaite 提供了换算因子表, 查此表 可获得该值。

$$
e=16(10 t / I)^{a}
$$

其中, $e$ 为标准月份最大蒸散量。标准月指日 数为 $30 \mathrm{~d}$, 日照时数为 $12 \mathrm{~h}$, 否则, 应进行修正以调 整到标准月; $t$ 为月均温 $; I$ 为温度指数。

$$
\begin{aligned}
& I=\sum i=\sum_{n=1}^{12}(t / 5)^{1.514} \\
& a=6.75 \times 10^{-7} \cdot I^{3}-7.71 \times 10^{-5} \cdot I^{2}+1.79 \times
\end{aligned}
$$$$
10^{-2} I+0.4924
$$

另外当观测点的纬度超过 $50^{\circ} \mathrm{N}$ 时, 采用 $50^{\circ} \mathrm{N}$ 的值; 当月均温超过 $26.5{ }^{\circ} \mathrm{C}$ 时采用一种表格, 由月 均温直接查得 $e$ 值。

陶诗言 (1949) 最早将 Thornthwaite 计算可能蒸 
散量的方法运用于中国气候区划中，该区划被认为 与中国的自然景观非常符合。Yim 和 Kira (1976) 在 研究朝鲜半岛的森林植被类型分布与气候的关系 时 利用 Thornthwaite 方法计算出了朝鲜半岛水分的 盈亏和湿润指数。张新时 (1989a；1989b) 根据我国 672 个国家气象观测站资料用 Thornthwaite 方法对中 国主要植被类型及其地理分布格局进行了较详尽的 分析,并提供了计算机计算与分类程序。Fang 和 Yoda（1990）也将该方法用于研究中国的水分平衡 与植被分布的关系。王义凤等 (1993) 应用 Thornthwaite的方法 对黄土高原 286 个气象台站的某些气 候指标进行了计算, 同时得到了每项气候指标的地 理回归模型，作出了各项气候指标的地区分布图，与 黄土高原地区植被的主要类型及其分布格局有密切 的关系。慈龙骏 (1994) 研究了全球变化对我国荒漠 化的影响 利用 Thornthwaite 的方法分析了二氧化碳 倍增、气温升高 $1.5 \sim 4.5{ }^{\circ} \mathrm{C}$ 时我国干旱区、半干旱 区及半湿润区的分布变化。 $\mathrm{Ma}$ 和 $\mathrm{Fu}(2003)$ 以 Thornthwaite 方法计算了1 $1951 \sim 1998$ 年的 160 个气 象台站的数据, 他认为, 我国北方地区的干燥度在增 加, 可西北地区的干燥度在减小, 北方地区干燥度增 加的主要原因是降水量的减少和蒸发量的增大。

联合国教科文组织 ( United nations educational , scientific, and cultural organization, UNESCO) 采用这 一方法的湿润指数标准划分了世界干旱区与半干旱 区的气候。1991 年 6 月联合国环境规划署 (United nations environment programme, UNEP) 在肯尼亚的内 罗华举行的 沙漠化评价与制图技术指导第三次会 议”上, 全球环境监测系统 (Global environment monitor system, GEMS)、全球资源信息数据库 (Global resource information database, GRID) 和沙漠化防治计划活动

中心 (Desert cure and prevention activity center, $\mathrm{DC} / \mathrm{PAS}$ ) 以湿润指数作为划分指标。他们所用的湿 润指数是采用 1977 年 Penman 公式的基础上修改后 的 Thornthwaite 公式, 计算降水量与可能蒸发量的比 例得出的参数。1994 年经过国际荒漠化公约政府 间谈判委员会的多次讨论，正式通过了《联合国关于 在发生严重干旱和/或荒漠化的国家特别是在非洲 防治荒漠化的公约》,此公约即利用 Thornthwaite 公 式计算湿润指数, 来定义荒漠化地区。该公约认为 干旱、半干旱和亚湿润干旱地区”是指年降水量与 潜在蒸散之比即湿润指数在 0.05 至 0.65 之间的地 区, 但不包括极区和副极区。慈龙骏等 (1997) 根据 联合国防治荒漠化公约的有关规定，运用 Thornth- waite 计算可能蒸散量的方法作出了第一张中国荒 漠化气候类型分布图, 首次确定了中国荒漠化的潜 在发生范围。

该方法的湿度气候类型划分比较合理，国际上 应用广泛，在气候分区中应用较多，特别适用于计算 逐月的资料。该方法在温暖地区可考虑降水，在寒 冷地区可考虑温度, 比较适于高纬度地区。

但是该方法对大气蒸发的极端情况反应不敏 感，且空气温度并不是蒸发的直接原因，所以作为主 要变量的温度不是蒸散率的最佳指标, 采用辐射值 可能提供更精确的结果 其次, 此方法中的在温度低 于 $0{ }^{\circ} \mathrm{C}$ 时蒸发停止作用的假设也不完全正确。由于 该方法是根据美国东部湿润气候条件下的渗透计观 测资料而建立,而我国地形复杂, 气候多变,所以用 此方法可能与我国的实际情况有较大出入。但相对 于 Penman 方法来说, 所需要的气候指标较少, 计算 相对简单。

\subsubsection{Holdridge 的可能蒸散量}

Holdrige 的计算方法 (Holdrige ,1947；1967) 是一 个通过温度来获得可能蒸散量的经验公式, 可能蒸 散量是温度的函数。公式如下：

$$
P E=58.93 \times A B T
$$

$A B T$ ( Annual biotemperature) 是年生物温度 $\left({ }^{\circ} \mathrm{C}\right)$, 生物温度是出现植物营养生长范围内的平均温度, 一般认为在 $0 \sim 30{ }^{\circ} \mathrm{C}$ 之间, 日均温低于 $0{ }^{\circ} \mathrm{C}$ 与高于 $30{ }^{\circ} \mathrm{C}$ 者均排除在外, 超过 $30{ }^{\circ} \mathrm{C}$ 的平均温度按 $30{ }^{\circ} \mathrm{C}$ 计算, 低于 $0{ }^{\circ} \mathrm{C}$ 的均按 $0{ }^{\circ} \mathrm{C}$ 计算。 $A B T$ 计算式如下：

$$
A B T=\frac{1}{12} \sum t_{i}
$$

张新时 (1993) 利用 Holdrige 方法对中国 700 多 个气候站计算的结果以生态信息系统进行了计算机 制图，绘制了中国蒸散率分布图。他认为 Holdrige 方法为基础的中国植被-气候分类提供了一个精度 基本上令人满意和便于应用的定量分析方法。他还 用此方法对大气中二氧化碳倍增条件下导致的气候 变化所引起的中国植被类型、分布界限和范围以及 生产力的演变趋势进行了预测。周筠珺等 (1996) 用 Thornthwaite 与 Holdridge 的 PE 指标对柴达木地区植 被-气候关系进行了分析,Thornthwaite 方法缩短的可 能蒸散量要比实际的小, 比较而言, Holdridge 的方法 较精细些,与植被的分布等有明显对应性。

在近年来研究全球变化对生态系统影响的评价 中 ,Holdrige 方法被广泛用于测试陆地生态系统分布 对模拟的气候变化的敏感程度及预测大气二氧化碳 
倍增条件下的植被和碳库变化格局。

Holdrige 方法所需气候指标简单, 计算简便, 生 态意义明确, 对应性强。缺点是此方法发展于中美 洲的热带地区，因而在中国的亚热带地区须进行局 部的调整; 年生物温度是从月平均温度而不是从更 短的时间尺度上计算的; 用 $30{ }^{\circ} \mathrm{C}$ 作为计算生物温度 的上限也不尽合理。

2.3 温度与降水计算的 de Martonne 干燥度

最简单的计算气候干燥度的方法就是利用温度 与降水这两个气候因子来计算干燥度, 此类方法主 要有 de Martonne 干燥度计算方法。

de Martonne(1926) 提出了一种简单的干燥度计 算方法：

$$
I_{d M}=\frac{P}{T+10}
$$

式中, $I_{d M}$ 即 de Martonne 干燥度, $P$ 为平均降水量 $(\mathrm{mm}), T$ 为平均温度值 $\left({ }^{\circ} \mathrm{C}\right)$ 。

干燥度值小于 10 ,表明严重干旱, 河流断流, 农 作物需要强制人工灌溉; 干燥度值在 $10 \sim 30$ 之间, 表明中等干旱, 河流暂时性有水, 流量中等, 植被类 型为草原, 干燥度大于 30 ,表明气候湿润, 河流常年 有水, 不断流, 并水量充足, 植被类型为森林。

$I_{d M}$ 因其计算简单, 指标明确, 与植被和水分对 应性强，所以经常用在气候区划上。Weck (1970) 根 据 $I_{d M}$ 建立了德国的气候模型。Botzan (1974) 利用 $I_{d M}$ 划分的水文特征提供了定义农业干旱的新指标。 Roberts (1993)估算了美国西南的亚利桑那州和中国 西北的乌鲁木齐两处沙漠地区的年干燥度, 并进行 了比较。Botzan (1998) 对 $I_{d M}$ 的计算进行了修改, 考 虑了水分的盈亏, 通过对拿帕盆地的研究, 他认为计 算结果与盆地的实际景观特征相符合。

$I_{d M}$ 更利于月干燥度的计算, 并且其计算简单, 但精确度不高, 比较适合于在大尺度的研究中应用。

\section{4 以积温来计算干燥度}

以积温来计算干燥度的方法主要有两种，一种 是以日温度大于或等于 $10{ }^{\circ} \mathrm{C}$ 的年积温计算的 Selianinov 干燥度计算方法; 另一种就是以月平均气 温高于 $5{ }^{\circ} \mathrm{C}$ 的年积温计算的 Kira 干湿度指数。

\subsubsection{Selianinov 干燥度}

谢良尼诺夫在 1937 年提出一经验公式, 利用温 度与降水量计算干燥度。原公式中的经验系数为 0.10 , 我国科学家根据我国的实际情况经过大量推 算 将 0.10 改为 0.16 (中国科学院自然区划工作委 员会 ,1959)。修正的谢良尼诺夫公式为：

\section{$K=0.16 \frac{\text { 全年 } \geq 10{ }^{\circ} \mathrm{C} \text { 的积温 }}{\text { 全年 } \geq 10{ }^{\circ} \mathrm{C} \text { 期间的降水量 }}$}

其中, $K$ 为干燥度。

中国科学院自然区划工作委员会 (1959)的中国 气候区划即根据干燥度进行的; 用 16.0 等值线作为 极干旱与干旱区的分界,此线与塔里木、柴达木盆 地、巴旦吉林和滕格里沙漠边缘一致,年降水量在 $60 \mathrm{~mm}$ 以下, 与我国荒漠景观大体吻合; 用 4.0 等值 线作为干旱与半干旱区的分界线,此线与旱作农业 西界相一致, 用 1.5 等值线作为干旱亚湿润与半干 旱区的分界指标，用 1.0 等值线作为湿润与干旱亚 湿润区的分界线, 此线与淮河秦岭一线基本一致(陈 建伟等, 1996)。黄朝迎(2003) 在研究黑河流域气候 变化对生态环境与自然植被影响时, 利用了谢良尼 诺夫的干燥度计算方法, 指出未来气温升高 $2{ }^{\circ} \mathrm{C}$, 而 降水量不变, 河西地区干燥度将增加 $0.5 \sim 1.1$, 且 越是干燥的地方,干燥度增加越大; 未来气温升高 $2{ }^{\circ} \mathrm{C}$, 而同时降水量增加 $10 \%$,干燥度则基本保持不 变 即降水量增加可大致抵消由气温升高而增加的 蒸发量。

谢氏干燥度计算方法相对容易获得所需数据， 以气象观测台站所记录的各个温度基点的年积温资 料, 不需作很大调整即可满足计算需要, 相对较为简 便可行，比较适合中高纬度地区。但谢氏干燥度的 计算方法是经验性的, 它假定一定的活动温度总和 可以代表一定的可能蒸发量, 这一假定缺乏物理意 义 是经验性的, 只在少数的地方经过初步验证, 未 必适用于全国。它只计算日均温持续在 $10{ }^{\circ} \mathrm{C}$ 以上 的时期, 而温度较低的季节仍有生物活动, 并且此时 的降水可以部分留在土壤中, 供温暖季节利用。所 以, 只计算 $10{ }^{\circ} \mathrm{C}$ 以上时期的温度, 在有些地方可能 造成很大的误差。

\subsubsection{Kira 干湿度指数}

$\operatorname{Kira}($ 吉良龙夫) 的干湿度指数是以降水与温暖 指数的比值来表示的 其计算公式为：

当 $W I=0 \sim 100{ }^{\circ} \mathrm{C} \quad$ 月 ${ }^{-1}$ 时,$k=P /(W I+20)$

当 $W I>100{ }^{\circ} \mathrm{C}$ 月 ${ }^{-1}$ 时, $k=2 P /(W I+140)$

式中, $P$ 为年降水量 ;温暖指数 $(W I)$ 是采用月平均 气温高于 $5{ }^{\circ} \mathrm{C}$ 的总和, 作为植物生长的热量条件, 即 :

$W I=\Sigma(t-5)(t$ :大于 5 的月均温 $)$

徐文铎(1983)利用吉良龙夫的热量指标和其修 正的干湿度指标, 分析了东北地区地带性植被建群 种和常见种的分布与水分、热量之间的定量关系, 为 
深入研究东北地区植被-气候分布规律, 开展植被区 划和探索植被-气候定量关系提供了科学依据。倪 健 (1997) 根据 Kira 以月平均气温 $5{ }^{\circ} \mathrm{C}$ 为界的热量指 数和干湿度指数概念, 提出了以月平均气温 $10{ }^{\circ} \mathrm{C}$ 为 界的生物热量指数, 并修正其干湿度指数为生物干 湿度指数。倪健等 $(1998 \mathrm{a} ; 1998 \mathrm{~b})$ 根据中国亚热带 常绿阔叶林区域的气象资料和植物分布资料，利用 Kira 的温暖指数和徐文铎的湿润指数计算了中国亚 热带常绿阔叶林的 112 个优势种及常见种的水热指 标值, 分析了树种分布与气候的关系。刘春迎 (1999)利用 Kira 的温度指数、寒冷指数、干湿度指数 公式对中国的 659 个气象站资料进行了计算与分区 统计 结果显示: 温暖指数和干湿度指数与中国植被 主要类型及其分布格局相关密切。

Kira 的计算方法简便，与植被的对应性好，值得 推广, 但这些指标是从东亚植被与气候的关系研究 中发展起来的, 其干湿度指数得自于雨温关系较单 一的夏雨型气候区, 并不适用于高寒地区, 在推广应 用时需根据雨温关系进行适当调整。

\section{5 辐射计算的 Budyko 干燥率}

1951 年布迪科和格里戈里耶夫合作,创立了 辐射干燥指数” 即干燥率 $(D)$, 在陆面充分湿润条 件下, 陆面最大可能蒸散量可以利用与确定水面蒸 发量相类似的方法计算, 即水面或湿润表面的蒸发 与按蒸发表面的温度计算出来的空气饱和差成正 比。一般借用热量平衡方程来确定蒸发面的温度, 从而可求蒸发力。表达式为 :

$$
D=R / L P
$$

式中, $R$ 为太阳净辐射, $L$ 为蒸发潜热, $P$ 为降水量。

Budyko(1974) 曾对 1600 个大致均匀分布在陆 地上的站点进行了辐射干燥度的计算, 作出了世界 分布图 结果与主要植被地带的配置颇为一致，再将 地面辐射差额值的增加列在一条垂直线上, 将辐射 干燥度作为水平线,获得了全球植被的分布图。 Uchijima 和 Seino(1985)根据布迪科的辐射平衡公式 和 IBP 研究计划对世界植被潜在净第一性生产力的 研究编制了 Chikugo 模型。Arora (2002)利用布迪科 的干燥率来估算气候变化对区域年径流量的影响， 他认为布迪科的干燥率可以通过降水量估算出年蒸 发量, 并根据降水量和热量的变化得到年径流量的 变化。Sankarasubramanian 和 Vogec (2002) 在研究美 国的年水文气象变化时利用布迪科的干燥率建立了 水分平衡模型。

布迪科的干燥率虽然计算简单, 但目前利用却
不多, 主要是由于其基本函数之一的太阳净辐射目 前缺乏足够参考的数据。

\section{3 几种干燥度指标范围}

由于各种干燥度指数的计算原理和公式各不相 同，所形成的指标体系也不一致，在气候分区中所对 应的指标见表 2 , 我们在实际应用时, 需要根据特定 的情况和不同的指标区别对待。

\section{4 各干燥度计算方法的使用比较}

Penman 公式法虽然计算合理,精确度高, 有较 好的物理学意义, 但计算复杂,所需的气象要素较 多，特别是在计算全国范围内的干燥度时，各气象站 点提供的气象数据不全, 如水汽压亏损值等在一般 台站没有记录, 致使无法计算。所以,Penman 公式 法在我国的使用有一定的限制。同样，作为布迪科 方法的重要计算数据一太阳净辐射 现阶段还不 能有效获取, 所以计算大范围的干燥度时也无法使 用。

Thornthwaite 公式法是根据美国东部湿润气候 条件下的渗透计观测资料而建立的,由于我国地形 复杂, 气候多变, 用此方法与我国的实际情况有较大 出入。慈龙骏等 (1997) 的区划中湿润地区过于偏 北，其面积占了我国总面积的近 $2 / 3$ 。其局部之所 以出现较大偏差, 主要原因之一是由于可能蒸散量 的计算结果误差较大。周晓东等 (2002) 也认为 Thornthwaite 公式计算的干燥度的等值线明显偏北， 而且在青藏高原地区, Thornthwaite 公式法作出的荒 漠化图未能反映青藏高原的水平地域分异。

吉良龙夫的干湿度指数适合于东亚的季风气 候，对我国森林植被的划分具有较好的指示作用，特 别是东北林区，但对西南部高山、亚高山地区指示性 不强, 对中国西北部干旱、半干旱区更不适应。

谢良尼诺夫公式法是一经验公式, 此方法计算 过程简单, 气候数据容易获取。中国属东亚季风环 流控制区，而干旱区位处温带，降水多集中于植物生 长期, 该方法经过我国科学家的修改以后, 比较适合 于我国干燥度的计算。

de Martonne 方法计算简单, 数据易得, 并且指标 体系的生态学意义明确, 特别是对农业生产有较好 的指导作用, 适合于大范围的干燥度指数的计算。 在我国的实际应用中看出，对我国的西北地区有较 好的利用价值。

Holdridge方法计算干燥度十分简便, 在近年来 
表 2 干燥度在气候类型中的划分范围

Table 2 Range of aridity index for different climatic types

\begin{tabular}{|c|c|c|c|c|c|c|}
\hline $\begin{array}{c}\text { 气候类型 } \\
\text { Climatic type }\end{array}$ & $\begin{array}{c}\text { UNEP 干燥度 } \\
\text { Aridity Index } \\
\text { of UNEP }\end{array}$ & $\begin{array}{c}P E \text { 计算干燥度 } \\
\text { Aridity index } \\
\text { of } P E\end{array}$ & $\begin{array}{c}\text { de Martonne } \\
\text { 干燥度 } \\
\text { Aridity index } \\
\text { of de Martonne }\end{array}$ & $\begin{array}{c}\text { 谢氏干燥度 } \\
\text { Aridity index } \\
\text { of Selianinov }\end{array}$ & $\begin{array}{c}\text { Kira 的干燥度 } \\
\text { Aridity index } \\
\text { of Kira }\end{array}$ & $\begin{array}{c}\text { 布迪科的干燥率 } \\
\text { Aridity index } \\
\text { of Budyko }\end{array}$ \\
\hline 湿润 Humid & $>0.75$ & $<1.00$ & $>30$ & $<1.0$ & $7.5 \sim 14.5$ & $<1.1$ \\
\hline 半湿润 Semi-humid & $0.65 \sim 0.75$ & $1 \sim 1.70$ & $10 \sim 30$ & $1 \sim 1.5$ & $4 \sim 7.5$ & $1.1 \sim 2.3$ \\
\hline 半干旱 Semi-arid & $0.20 \sim 0.45(\sim 0.65)$ & $1.7 \sim 3$ & $10 \sim 30$ & $1.5 \sim 4$ & $2.5 \sim 4$ & $2.3 \sim 3.4$ \\
\hline 干旱 Arid & $0.05 \sim 0.20$ & $3 \sim 8$ & $<10$ & $4 \sim 16$ & $<2.5$ & $3.4 \sim 10$ \\
\hline 极干旱 Hyper-arid & $<0.05$ & $>8.0$ & $<10$ & $>16$ & & $>10$ \\
\hline 备注 Note & $\begin{array}{c}\text { Le Houerou , } \\
1996\end{array}$ & 陈建伟等 ,1996 & Botzan , 1998 & 张煜星，1998 & 刘春迎 ,1999 & 黄培祐 ,2002 \\
\hline
\end{tabular}

研究全球变化对生态系统的影响评价中发挥了极大 的作用，对于预测二氧化碳倍增条件下局部及全球 大尺度上植被的变化格局十分有用。由于我国月均 温没有高于 $30{ }^{\circ} \mathrm{C}$ 上限的地区, 所以对于我国来说, 此方法也比较适合。

\section{5 研究展望}

在比较分析了上述多种干燥度指数之后, 我们 需要进一步利用我国的气象数据, 计算几种较适用 的干燥度指数, 分析其空间分布特征, 对所得干燥度 指数空间和时间序列分布图进行比较, 以从中发现 它们之间的异同, 寻求各种干燥度指数之间的可比 性。

利用谢良尼诺夫, de Martonne 和 Holdridge 这几 种干燥度指数, 可以分析过去 50 年里我国干湿气候 界线的波动，确定我国现在的干湿气候区域，并探索 过去 50 年来全国 ,特别是北方地区干湿气候变化的 趋势及其与全球气候变化的关系, 以此预测未来气 候变化可能对我国干旱化趋势和特征的影响，以及 由此所引起的植被变化和土地荒漠化等的变化趋 势, 为制定应对的策略奠定科学基础。

\section{参 考 文 献}

Arora, V. K. 2002. The use of the aridity index to assess climate change effect on annual runoff. Journal of Hydrology, 265: 164 $\sim 177$.

Botzan, M. G. 1974. Consideratii asupra indicilor climatici pentru proiectarea irigatiilor (Concerns on climatic indexes for irrigation design). Hidrotehnica, 19: $41 \sim 48$.

Botzan, M. G. 1998. Modified de Martonne aridity index: application to the Napa Basin, California. Physical Geography, 19: 55 $\sim 70$.

Budyko, M. I. 1974. Climate and life. New York: Academic Press.

Chen, J. W. (陈建伟) \& Y. X. Zhang (张煜星). 1996. Discussion on relation between humidity index and aridity degree. Journal of Desert Research (中国沙漠), 16: 79 82. (in Chi- nese with English abstract)

Ci, L. J. (慈龙骏). 1994. The influence of global change on desertification in China. Journal of Natural Resources (自然资源 学报), 9: 189 303. (in Chinese with English abstract)

Ci, L. J. (慈龙骏) \& B. Wu (吴波). 1997. Climatic type division and the potential extent determination of desertification in China. Journal of Desert Research (中国沙漠), 6: 107 111. (in Chinese with English abstract)

Committee of Physical Regionalization of the Chinese Academy of Sciecnes(中国科学院自然区划工作委员会). 1959. Synthetic physical regionalization of China (Draft). Beijing: Science Press. (in Chinese)

Dzerdzeevskii, B. L. 1958. On some climatological problems and microclimatological studies of arid and semi-arid regions in U.S. S.R. Proc. Canberra Symposium of Arid Zone Research, Paris: UNESCO Publication. 315 325 .

Fang, J. Y. \& K. Yoda. 1990. Climate and vegetation in China. III . Water balance and distribution of vegetation. Ecological Research, 5: $9 \sim 23$.

Geng, K. H. (耿宽宏). 1986. Climate of Chinese desert. Beijing: Science Press. (in Chinese)

Henry, N. L. 1996. Climate change, drought and desertification. Journal of Arid Environments, 34: 133 185.

Hess, T. M. 1998. Trends in reference evapotranspiration in the North East Arid Zone of Nigeria, 1961 - 90. Journal of Arid Environments, 38: $99 \sim 115$.

Holdridge, L. R. 1947. Determination of world plant formation from simple climatic data. Science, 105: $367 \sim 368$.

Holdridge, L. R. 1967. Life zone ecology. San Jose, Costa Rica: Tropical Science Center.

Huang, C. Y. (黄朝迎). 2003. A diagnostic analysis for the impact of climate change on eco-environment and natural vegetation in the Heihe River valley. Climatic and Environmental Research (气候与环境研究), 8: 84 90. (in Chinese with English abstract)

Huang, P. Y. (黄培祐). 2002. Excused irrigation vegetation in arid area and its restoration. Beijing: Science Press. $6 \sim 13$. (in Chinese)

Le Houerou, H. N. 1996. Climate change, drought and desertification. Journal of Arid Environments, 34: $133 \sim 185$.

Liu, C. Y. (刘春迎). 1999. The application of Kira's indices to the study of vegetation-climatic interaction in China. Acta Phytoecologica Sinica (植 物生态学报), 23: 125 138. (in Chinese with English abstract)

Liu, Q. J. , A. Kondoh \& N. Takeuchi. 1998. Study of changes in life zone distribution in north-east China by climate-vegetation classification. Ecological Classification, 13: $355 \sim 365$.

Ma, Z. G. \& C. B. Fu. 2003. Interannual characteristics of the surface hydrological variables over the arid and semi-arid areas of northern China. 
Global and Planetary Change, 37: 189 200 .

$\mathrm{Ni}, \mathrm{J}$. (倪健). 1998. Indexes of vegetation-climate classification and its applications. Chinese Journal of Ecology (生态学杂志), 17: 33 44. (in Chinese with English abstract)

Ni，J. (倪健) \& Y. C. Song (宋永昌). 1998a. A discussion on the relationship between Penman's indices and distribution of some tree species of subtropical evergreen broadleaved forest in China. Acta Botanica Sinica (植物学报), 40: 647 656. (in Chinese with English abstract)

Ni, J. (倪健) \& Y. C. Song (宋永昌). 1998b. Relationship between Kira's index and distribution of dominants and companions of subtropical evergreen broadleaved forest in China. Acta Ecologica Sinica (生态学报), 18: $248 \sim 262$. (in Chinese with English abstract)

Ni, J. (倪健). 1997. Development of Kira's indices and its application to vegetation-climate interaction study of China. Chinese Journal of Applied Ecology (应用生态学报), 8: $161 \sim 170$. (in Chinese with English abstract)

Penman, H. L. 1956. Estimating evaporation. Transaction of American Union, 37: $43 \sim 50$.

Penman, H. L. 1948. Natural evaporation from open water, bare soil, and grass. Proceedings Royal Society Series A, 193: 454 465 .

Ren, F. M. (任福民) \& J. E. Shi (史久恩). 1995. Characteristics of annual rainfall in arid and semi-arid region of China. Quarterly Journal of Applied Meteorology (应用气象学报), 6: 502 504. (in Chinese with English abstract)

Roberts, B. R. 1993. Water management in desert environments. Berlin: Springer-Verlag.

Sankarasubramanian, A. \& R. M. Vogel. 2002. Annual hydroclimatology of the United States. Water Resources Research, 38: $19-1 \sim 19-12$, doi 10.1029/2001 WR000619.

Shang, Z. B., Q. Gao \& D. A. Yang. 2001a. Digital ecological model and case study on China water condition. Ecological Modelling, 139: 235 $\sim 252$.

Shang, Z. B., Q. Gao \& D. A. Yang. 2001b. The climate information system of China. Climate Research, 18: 229 247.

Tao, S. Y. (陶诗言). 1949. The analyses of the required water and the new classification of climate in China. Acta Meteorologica Sinica (气象学 报), 20: $43 \sim 50$. (in Chinese)

Thornthwaite, C. W. 1948. An approach toward a rational classification of climate. Geographical Review, 38: $57 \sim 94$.

Uchijima, Z. \& H. Seino. 1985. Agroclimatic evaluation of net primary producticity of natural vegetation. (1) Chikugo model for evaluating primary productivity. Journal of Agricultural Meteorology, 40: $343 \sim 352$.
Wang, Y. F. (王义风) \& X. M. Xiao (肖向明) . 1993. Climatic gradient of main vegetation types in the loess plateau region. Acta Botanica Sinica (植物学报), 35: $291 \sim 299$. (in Chinese with English abstract)

Weck, J. 1970. An improved CVP-index for the delimitation of the potential productivity zones of forest lands of India. Indian Forester, 96: 565 572 .

Xi, C. F. (席承藩)， B. J. Qiu (丘宝剑) \& J. M. Zhang (张俊民) . 1984. Chinese demarcation of nature. Beijing: Science Press. (in Chinese)

Xu, W. D. (徐文铎).1983. A study on the relation between keystone and common species distribution and hydrothermal factors in Northeast China. Acta Botanica Sinica (植物学报), 25: $264 \sim 273$. (in Chinese with English abstract)

Yim, Y. J. \& T. Kira. 1976. Distribution of forest vegetation and climate in the Korean Peninsula II . Distribution of climatic humidity/aridity. Japanese Journal of Ecology, 26: $157 \sim 164$.

Zhang, X. S. (张新时). 1989a. The potential evapotranspiration $(P E)$ index for vegetation and vegetation-climatic classification (1) - an introduction of main methods and PEP program. Acta Phytoecologica et Geobotanica Sinica (植物生态学与地植物学学报), 13: 1 9. (in Chinese with English abstract)

Zhang, X. S. (张新时). 1989b. The potential evapotranspiration ( $P E$ ) index for vegetation and vegetation-climatic classification $(2)-$ an introduction of main methods and PEP program. Acta Phytoecologica et Geobotanica Sinica (植物生态学与地植物学学报), 13: 197 207. (in Chinese with English abstract)

Zhang, X. S. (张新时). 1993. A vegetation-climate classification system for global change studies in China. Quaternary Sciences (第四纪研究), 2: $157 \sim 169$. (in Chinese with English abstract)

Zhang, Y. X. (张煜星). 1998. Distribution of climatic type of Chinese desertification. Arid Zone Research (干旱区研究), 15: 46 50. (in Chinese with English abstract)

Zhou, X. D. (周晓东), Q. J. Zhu (朱启疆), Z. P. Sun (孙中平) \& R. Sun (孙睿). 2002. Preliminary study on regional desertification climate in China. Journal of Natural Disasters (自然灾害学报), 11: 125 $\sim 131$. (in Chinese with English abstract)

Zhou, Y. J. (周筠珺) \& L. H. Zhou (周立华). 1996. Preliminary analysis for vegetation-climate relation in Caidam District by the potential evapotranspiration $(P E)$ indexes of Thornthwaite and Holdridge. Arid Zone Research (干旱区研究), 13: 46 51. (in Chinese with English abstract) 